\title{
Investigation of hybrid gain-clamped Raman-fiberamplifier/EDFA utilizing pump reuse technique
}

\begin{abstract}
We investigate the performance of hybrid gainclamped Raman-fiber-amplifier (RFA)/Erbium-doped fiber amplifier (EDFA) with respect to the conventional gain-clamped Raman fiber amplifier. The hybrid gain-clamped amplifier is constructed in two configurations: RFA/EDFA and EDFA/RFA. The conventional gain-clamped RFA produces the lowest gain variation, the lowest noise figure and the highest critical power amongst three amplifier configurations. By cascading the RFA and EDFA in series, the hybrid gain-clamped amplifiers suffer performance degradation due to the unique characteristics of RFA and EDFA. The noise figure degradation is strongly influenced by the performance of the first stage amplifier and the RFA/EDFA configuration produces the highest noise figure. On the other hand, the EDFA/RFA configuration experiences fastest saturation due to the pump depletion in the RFA. (@ 2008 by Astro Ltd., Published exclusively by WILEY-VCH Verlag $\mathrm{GmbH} \& \mathrm{Co} . \mathrm{KGaA}$ )
\end{abstract}

Keyword: Raman fiber amplifier, Erbium-doped fiber amplifier, Gain-clamped 